\title{
Pseudospin-electron model in the self-consistent gaussian fluctuation approximation
}

\author{
I.V.Stasyuk, K.V.Tabunshchyk \\ Institute for Condensed Matter Physics \\ of the National Academy of Sciences of Ukraine, \\ 1 Svientsitskii Str., 79011 Lviv, Ukraine
}

Received October 24, 2000

\begin{abstract}
Pseudospin-electron model with an effective many-body interaction between pseudospins via conducting electrons is investigated within the generalized random phase approximation scheme with the self-consistent inclusion of mean field type contributions (coming from the effective pseudospin interaction) as well as gaussian fluctuations of the mean field (which makes it possible to obtain more accurate results in the vicinity of the critical points). Using the approach proposed here, the expressions are obtained for the pseudospin correlation function, for pseudospin mean value, as well as for the grand canonical potential.
\end{abstract}

Key words: pseudospin-electron model, local anharmonicity, gaussian fluctuations

PACS: $71.10 . F d, 71.38 .+i, 77.80 . B h, 63.20 . R y$

\section{Introduction}

Pseudospin-electron model (PEM) considered herein was proposed to describe the interaction of the conducting electrons in metals or in semimetals with some two level subsystem represented by pseudospins (e.g. anharmonic vibrations of the apex oxygen ions in $\mathrm{YBaCuO}$-type crystals [1]), as well as the proton-electron interaction in the molecular and crystalline systems with hydrogen bonds [2].

The corresponding Hamiltonian has the following form

$$
H=H_{0}+\sum_{i j \sigma} t_{i j} c_{i \sigma}^{+} c_{j \sigma}, \quad H_{0}=\sum_{i}\left\{-\mu \sum_{\sigma} n_{i \sigma}+g \sum_{\sigma} n_{i \sigma} S_{i}^{z}-h S_{i}^{z}\right\},
$$

and includes the terms describing electron transfer $\left(\sim t_{i j}\right)$, energy of the subsystem of pseudospins placed in longitudinal field $(\sim h)$ and local interaction $(\sim g)$ of conducting electrons with pseudospins. 
Hamiltonian (1) can be transformed, after some simplification, into the Hamiltonian of the electron subsystem of binary alloy type model as well as the FalicovKimball model. The main difference between these models lies in the way how an averaging procedure is performed (thermal statistical averaging in the case of PEM and Falicov-Kimball model, configurational averaging for binary alloy) $[3,4]$. This Hamiltonian is also invariant with respect to the transformation $\mu \rightarrow-\mu, h \rightarrow 2 g-h$, $n \rightarrow 2-n, S^{z} \rightarrow-S^{z}$. It allows us to use (1) for the description of a hole-pseudospin system as well.

In the previous papers [5-7], a self-consistent scheme was proposed for the calculation of mean values of pseudospin and electron number operators, grand canonical potential as well as correlation functions of simplified PEM. The main idea of the approach was based on the GRPA scheme [8] with the inclusion of the mean field type contributions coming from the effective pseudospin interactions via conducting electrons [5]. On the basis of this self-consistent mean field type approximation, the energy spectrum, thermodynamics of phase transitions, the possibility of phase separations as well as the appearance of the chess-board phase were investigated. It was shown that: in the $\mu=$ const regime (when it is supposed that the electron states of the other structure elements, which are not included explicitly into the PEM, play a role of a thermostat, that ensures a fixed value of the chemical potential $\mu$ ), the interaction between the electron and pseudospin subsystems leads to the possibility of either first or second order phase transitions between different uniform phases (bistability effect) as well as between the uniform and the chess-board ones [5,7]; in the regime $n=$ const (this situation is more customary at the consideration of electron systems and means that the chemical potential being the function of $T, h$ etc. depends now on the electron concentration), an instability with respect to the phase separation in the electron and pseudospin subsystems can take place [5,7].

An approach, that takes into account only mean field type contributions, is reasonable only when deviations from the average values are small or, in other words, in the area where the effects of fluctuations are unimportant. Therefore, the previously proposed method [5,7] makes it possible to obtain accurate results outside the region of the critical point.

In the vicinity of the critical point the effects of the mean field fluctuations become significant. Hence, to improve the description of the thermodynamics of our system we should correct the approach by taking into account the contribution of the fluctuations of the self-consistent field of pseudospins.

For this purpose, we construct a consistent scheme (using the diagram method), to calculate the pseudospin operator mean value, the grand canonical potential as well as the pseudospin correlation function of simplified PEM, which allows us to take into account the gaussian fluctuations of the self-consistent mean field. Rootmean-square (r.m.s.) fluctuations of the field are calculated in a self-consistent way. Within the high density expansion method the r.m.s. of gaussian fluctuations of the molecular field were previously considered in the case of spin models $[9,10]$. In the present paper we generalize this scheme on the pseudospin-electron model (where we have an effective many-body interaction between pseudospins via conducting 
electrons). Using the the scheme proposed here, the expression for the grand canonical potential as well as a set of equations for the pseudospin mean value and r.m.s. fluctuations parameter are obtained.

\section{Mean field approximation}

We perform the calculations in the strong coupling case $(g \gg t)$ using singlesite states as the basic ones. We rewrite the initial Hamiltonian of the simplified $\mathrm{PEM}$ in the second quantized form using projecting electron annihilation (creation) operators [5-7] $a_{i \sigma}=c_{i \sigma} P_{i}^{+}, \tilde{a}_{i \sigma}=c_{i \sigma} P_{i}^{-}\left(P_{i}^{ \pm}=\frac{1}{2} \pm S_{i}^{z}\right)$ acting at a site with the certain pseudospin orientation:

$$
H=H_{0}+H_{\mathrm{int}}=\sum_{i \sigma}\left\{\varepsilon n_{i \sigma}+\tilde{\varepsilon} \tilde{n}_{i \sigma}-\frac{h}{2} S_{i}^{z}\right\}+\sum_{i j \sigma} t_{i j}\left(a_{i \sigma}^{+} a_{j \sigma}+a_{i \sigma}^{+} \tilde{a}_{j \sigma}+\tilde{a}_{i \sigma}^{+} a_{j \sigma}+\tilde{a}_{i \sigma}^{+} \tilde{a}_{j \sigma}\right),
$$

where $\varepsilon=-\mu+g / 2, \quad \tilde{\varepsilon}=-\mu-g / 2$ are the energies of the single-site states.

Expansion of the calculated quantities in terms of the electron transfer leads to an infinite series of terms containing the averages of the $T$-products of the $a_{i \sigma}, \tilde{a}_{i \sigma}$ operators. The evaluation of such averages is made using the corresponding Wick's theorem. The results are expressed in terms of the products of the nonperturbed Green's functions and the averages of the products of projection operators $P_{i}^{ \pm}$which are calculated by means of the semi-invariant expansion [5].

From such an infinite series we are summing up a certain partial sum of diagrams (in the spirit of the traditional mean field approach [5]) characterized by the inclusion, in all basic semi-invariants, of the mean field type contributions (i.e., loop fragments) coming from the effective many-body interaction between pseudospins via conducting electrons.

The corresponding diagram series for pseudospin mean value has the form:

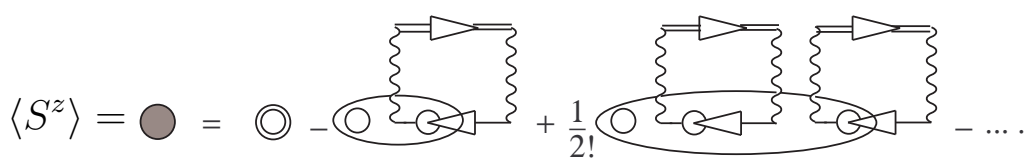

Here we use the following diagram notations: $\bigcirc-S^{z}, \longrightarrow--g_{i}\left(\omega_{n}\right)$, wavy line is the Fourier transform of the hopping parameter $t_{\boldsymbol{k}}$. Basic semi-invariants are represented by ovals and contain the $\delta$-symbols on site indexes.

$$
\left(\mathrm{O}=\left\langle S^{z}\right\rangle_{0}=b(h)=\frac{\mathrm{Sp}\left(S^{z} \mathrm{e}^{-\beta H_{0}}\right)}{\mathrm{Sp}\left(\mathrm{e}^{-\beta H_{0}}\right)}, \quad \bigcirc \mathrm{O}=\left\langle S^{z} S^{z}\right\rangle_{0}^{\mathrm{c}}=\frac{\partial b(h)}{\partial \beta h} .\right.
$$

Nonperturbed electron Green's function is equal to

$$
\longrightarrow{ }^{-}=g\left(\omega_{n}\right)=\left\langle g_{i}\left(\omega_{n}\right)\right\rangle, \quad g_{i}\left(\omega_{n}\right)=\frac{P_{i}^{+}}{\mathrm{i} \omega_{n}-\varepsilon}+\frac{P_{i}^{-}}{\mathrm{i} \omega_{n}-\tilde{\varepsilon}} .
$$

The full single-electron Green's function is

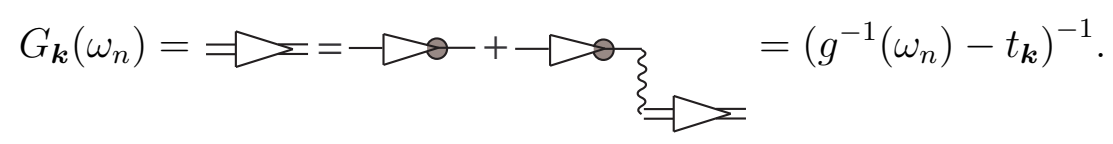


It determines the electron spectrum.

$$
\varepsilon_{\mathrm{I}, \mathrm{II}}\left(t_{\boldsymbol{k}}\right)=\frac{1}{2}\left(2 E_{0}+t_{\boldsymbol{k}}\right) \pm \frac{1}{2} \sqrt{g^{2}+4 t_{\boldsymbol{k}}\left\langle S^{z}\right\rangle g+t_{\boldsymbol{k}}^{2}} .
$$

This spectrum was investigated in detail in [5]

Expression for the loop fragment of diagram has the following form

$$
\sum_{Q}=\frac{2}{N} \sum_{n, \boldsymbol{k}} \frac{t_{\boldsymbol{k}}^{2}}{g^{-1}\left(\omega_{n}\right)-t_{\boldsymbol{k}}}\left(\frac{P_{i}^{+}}{\mathrm{i} \omega_{n}-\varepsilon}+\frac{P_{i}^{-}}{\mathrm{i} \omega_{n}-\tilde{\varepsilon}}\right)=\beta\left(\alpha_{1} P_{i}^{+}+\alpha_{2} P_{i}^{-}\right) .
$$

It creates an internal effective self-consistent field acting on the pseudospin.

Now, we can introduce the following mean field Hamiltonian:

$$
H_{\mathrm{MF}}=\sum_{i}\left\{\varepsilon\left(n_{i \uparrow}+n_{i \downarrow}\right)+\tilde{\varepsilon}\left(\tilde{n}_{i \uparrow}+\tilde{n}_{i \downarrow}\right)-y S_{i}^{z}\right\}
$$

where $y=h+\alpha_{2}-\alpha_{1}$ is a mentioned effective field. Summation of the diagram series (3) is equivalent to the averaging with the Hamiltonian (9). The result can be expressed in the form

$$
\begin{aligned}
\left\langle S_{l}^{z}\right\rangle & =b(y), \\
b(y) & =\frac{1}{2} \tanh \left\{\frac{\beta}{2} y+\ln \frac{1+\mathrm{e}^{-\beta \varepsilon}}{1+\mathrm{e}^{-\beta \tilde{\varepsilon}}}\right\} .
\end{aligned}
$$

Diagram equation for the pseudospin correlation functions $\left\langle S^{z} S^{z}\right\rangle_{\boldsymbol{q}}$ (obtained within the framework of GRPA with the insertion of the mean field type contributions into all zero-order semi-invariants) is as follows [5]:

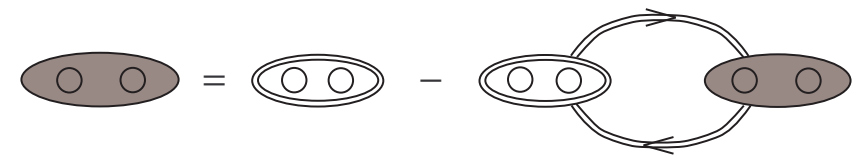

This equation differs from the one for the Ising model in RPA by the replacement of the exchange interaction by the electron loop $\Pi_{q}=$ an effective many-body interaction between pseudospins via conducting electrons:

$$
\Pi_{q}=\frac{2}{N} \sum_{n, \boldsymbol{k}} \Lambda_{n}^{2} \tilde{t}_{n}(\boldsymbol{k}) \tilde{t}_{n}(\boldsymbol{k}+\boldsymbol{q}), \quad \Lambda_{n}=\frac{g}{\left(\mathrm{i} \omega_{n}+\mu\right)^{2}-g^{2} / 4}, \quad \tilde{t}_{n}(\boldsymbol{k})=\frac{t_{\boldsymbol{k}}}{\left(1-g_{n} t_{\boldsymbol{k}}\right)}
$$

The first term in (12) is the second-order semi-invariants renormalized due to the inclusion of "single-tail" parts, and is thus calculated by means of $H_{\mathrm{MF}}$ :

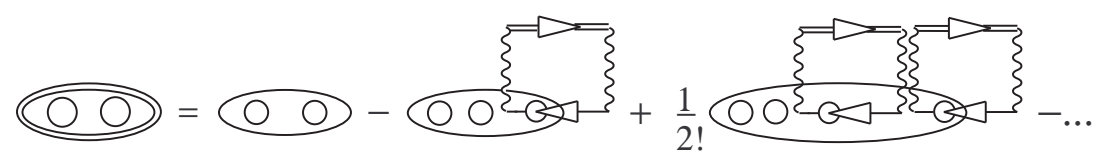


Finally, the solution of the equation (12) in the analytical form is:

$$
\left\langle S^{z} S^{z}\right\rangle_{\boldsymbol{q}}=\frac{1 / 4-\left\langle S^{z}\right\rangle^{2}}{1+\frac{2}{N} \sum_{n, \boldsymbol{k}} \Lambda_{n}^{2} \tilde{t}_{n}(\boldsymbol{k}) \tilde{t}_{n}(\boldsymbol{k}+\boldsymbol{q})\left(1 / 4-\left\langle S^{z}\right\rangle^{2}\right)}
$$

and is different from zero only in a static case $\left(\omega_{n}=0\right)$ (this is due to the fact that the pseudospin operator commutes with the Hamiltonian).

In the same approximation, the grand canonical potential has the diagrammatic representation:

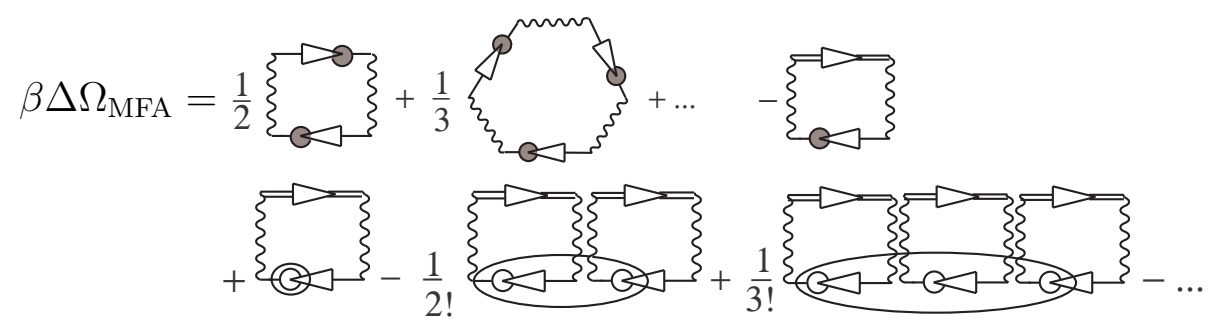

The corresponding analytical expression is:

$$
\Omega_{\mathrm{MFA}}=-\frac{2}{N \beta} \sum_{n, \boldsymbol{k}} \ln \left(1-t_{\boldsymbol{k}} g\left(\omega_{n}\right)\right)-\frac{2}{N \beta} \sum_{n, \boldsymbol{k}} \frac{\left.g\left(\omega_{n}\right)\right) t_{\boldsymbol{k}}^{2}}{\left.g^{-1}\left(\omega_{n}\right)\right)-t_{\boldsymbol{k}}}-\frac{1}{\beta} \ln \operatorname{Sp}\left(\mathrm{e}^{-\beta H_{\mathrm{MF}}}\right) .
$$

All quantities can be derived from the grand canonical potential by differentiating

$$
\frac{\mathrm{d} \Omega_{\mathrm{MFA}}}{\mathrm{d}(-h)}=\left\langle S^{z}\right\rangle, \quad \frac{\mathrm{d}\left\langle S^{z}\right\rangle}{\mathrm{d}(\beta h)}=\left\langle S^{z} S^{z}\right\rangle_{\boldsymbol{q}=0}
$$

that shows the thermodynamical consistence of the proposed approximation [5].

\section{Self-consistent gaussian fluctuation approximation}

In constructing a higher order approximation, we use MFA as the zero-order one. This means that all "single-tail" parts of diagrams are already summed up and all semi-invariant are calculated using the distribution with the Hamiltonian $H_{\mathrm{MF}}$ (9). We represent this graphically by thick ovals:

$$
\text { DIDD }=\text { DIID }- \text { 运 }
$$

As the simplest approximation that goes beyond the MFA, we use the approach that takes into account the so-called "double-tail" diagrams (such an approximation was used in $[9,10]$ where the magnetization of the ordinary Ising model was considered). The corresponding diagram series for the pseudospin mean value is:

$$
\left\langle S^{z}\right\rangle=0=0+\frac{1}{2} \frac{1}{1}, 00+\frac{1}{2} \frac{1}{2} \text {, }
$$


The diagram equation for pseudospin correlator $\left\langle S^{z} S^{z}\right\rangle_{\boldsymbol{q}}$ within the approximation developed here is given by (12), but now zero-order correlators are renormalized, also, due to the "double-tail" parts, and thus the corresponding diagram series is:

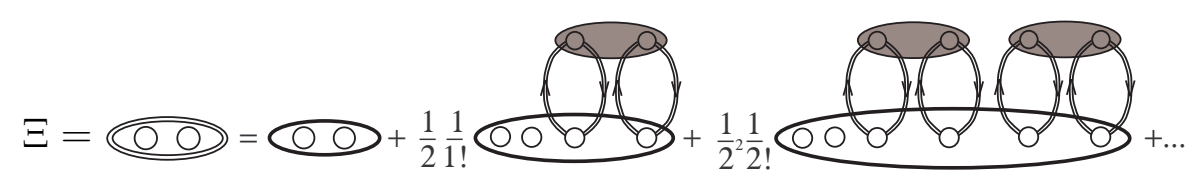

The contribution, which correspond to the "double-tail" fragment of the diagram, can be written in the following analytical form (using the notation (13)):

$$
\begin{gathered}
X=\int_{N^{3}} \sum_{n, n^{\prime}} \sum_{\boldsymbol{k}, \boldsymbol{k}^{\prime}} \sum_{\boldsymbol{q}} \Lambda_{n}^{2} \tilde{t}_{n}(\boldsymbol{k}) \tilde{t}_{n}(\boldsymbol{k}-\boldsymbol{q})\left\langle S^{z} S^{z}\right\rangle_{\boldsymbol{q}} \Lambda_{n^{\prime}}^{2} \tilde{t}_{n^{\prime}}\left(\boldsymbol{k}^{\prime}\right) \tilde{t}_{n^{\prime}}\left(\boldsymbol{k}^{\prime}+\boldsymbol{q}\right), \\
\left\langle S^{z} S^{z}\right\rangle_{\boldsymbol{q}}=\frac{\Xi}{1+\frac{2}{N} \sum_{n, \boldsymbol{k}} \Lambda_{n}^{2} \tilde{t}_{n}(\boldsymbol{k}) \tilde{t}_{n}(\boldsymbol{k}+\boldsymbol{q}) \Xi} .
\end{gathered}
$$

Since the pseudospin correlator (23) is frequency independent, in the expression (22) we have two independent sums over internal Matsubara frequencies that allows one (using decomposition into simple fractions) to sum over all internal frequencies:

$$
\begin{aligned}
\frac{2}{N} \sum_{n, \boldsymbol{k}, \boldsymbol{k}^{\prime}} \Lambda_{n}^{2} \tilde{t}_{n}(\boldsymbol{k}) \tilde{t}_{n}\left(\boldsymbol{k}^{\prime}\right)= & \frac{2 \beta}{N} \sum_{\boldsymbol{k}, \boldsymbol{k}^{\prime}} \frac{t_{\boldsymbol{k}} t_{\boldsymbol{k}^{\prime}}(\varepsilon-\tilde{\varepsilon})^{2}}{\left[\varepsilon_{\mathrm{I}}\left(t_{\boldsymbol{k}}\right)-\varepsilon_{\mathrm{II}}\left(t_{\boldsymbol{k}}\right)\right]\left[\varepsilon_{\mathrm{I}}\left(t_{\boldsymbol{k}^{\prime}}\right)-\varepsilon_{\mathrm{II}}\left(t_{\boldsymbol{k}^{\prime}}\right)\right]} \\
& \times\left\{\frac{n\left[\varepsilon_{\mathrm{I}}\left(t_{\boldsymbol{k}}\right)\right]-n\left[\varepsilon_{\mathrm{I}}\left(t_{\boldsymbol{k}^{\prime}}\right)\right]}{\varepsilon_{\mathrm{I}}\left(t_{\boldsymbol{k}}\right)-\varepsilon_{\mathrm{I}}\left(t_{\boldsymbol{k}^{\prime}}\right)}+\frac{n\left[\varepsilon_{\mathrm{II}}\left(t_{\boldsymbol{k}}\right)\right]-n\left[\varepsilon_{\mathrm{II}}\left(t_{\boldsymbol{k}^{\prime}}\right)\right]}{\varepsilon_{\mathrm{II}}\left(t_{\boldsymbol{k}}\right)-\varepsilon_{\mathrm{II}}\left(t_{\boldsymbol{k}^{\prime}}\right)}\right. \\
& \left.-\frac{n\left[\varepsilon_{\mathrm{I}}\left(t_{\boldsymbol{k}}\right)\right]-n\left[\varepsilon_{\mathrm{II}}\left(t_{\boldsymbol{k}^{\prime}}\right)\right]}{\varepsilon_{\mathrm{I}}\left(t_{\boldsymbol{k}}\right)-\varepsilon_{\mathrm{II}}\left(t_{\boldsymbol{k}^{\prime}}\right)}-\frac{n\left[\varepsilon_{\mathrm{II}}\left(t_{\boldsymbol{k}}\right)\right]-n\left[\varepsilon_{\mathrm{I}}\left(t_{\boldsymbol{k}^{\prime}}\right)\right]}{\varepsilon_{\mathrm{II}}\left(t_{\boldsymbol{k}}\right)-\varepsilon_{\mathrm{I}}\left(t_{\boldsymbol{k}^{\prime}}\right)}\right\} .
\end{aligned}
$$

Here $n(\varepsilon)=\frac{1}{1+\mathrm{e}^{\beta \varepsilon}}$ is a Fermi distribution.

Let us now return to the problem of summation of the diagram series (20) and (21). By means of the procedure described in [10], and using notations (11), (22) we can write:

$$
\begin{aligned}
\left\langle S^{z}\right\rangle & =b(y)+\frac{1}{1 !} b(y)^{[2]} \frac{X}{2}+\frac{1}{2 !} b(y)^{[4]}\left(\frac{X}{2}\right)^{2}+\frac{1}{3 !} b(y)^{[6]}\left(\frac{X}{2}\right)^{3}+\cdots \\
& =\frac{1}{\sqrt{2 \pi X}} \int_{-\infty}^{+\infty} \exp \left(-\frac{\xi^{2}}{2 X}\right) b(y+\xi) \mathrm{d} \xi \\
\Xi & =b(y)^{[1]}+\frac{1}{1 !} b(y)^{[3]} \frac{X}{2}+\frac{1}{2 !} b(y)^{[5]}\left(\frac{X}{2}\right)^{2}+\frac{1}{3 !} b(y)^{[7]}\left(\frac{X}{2}\right)^{3}+\cdots \\
& =\frac{1}{X \sqrt{2 \pi X}} \int_{-\infty}^{+\infty} \exp \left(-\frac{\xi^{2}}{2 X}\right) \xi b(y+\xi) \mathrm{d} \xi .
\end{aligned}
$$

Therefore, the contribution of diagram series with "double-tail" parts corresponds to the average with the Gaussian distribution where $X$ can be interpreted as the 
root-mean-square (r.m.s.) fluctuation of the mean field around the mean value of $y$. Thus we obtain a self-consistent set of equations (25), (22) for pseudospin mean value and r.m.s. fluctuations parameter.

The diagram series for the grand canonical potential within the approximation accepted here is:

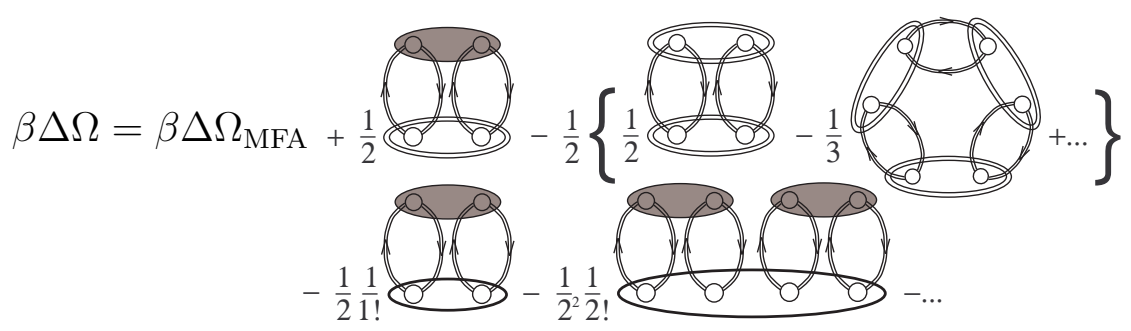

The grand canonical potential written in this form satisfies the stationary conditions:

$$
\frac{\mathrm{d} \Omega}{\mathrm{d}\left\langle S^{z}\right\rangle}=0, \quad \frac{\mathrm{d} \Omega}{\mathrm{d} X}=0
$$

which are equivalent to the equations (25), (22). The consistency of the approximations used for the pseudospin mean value, pseudospin correlation function and grand canonical potential can be checked explicitly using the relations:

$$
\frac{\mathrm{d} \Omega}{\mathrm{d}(-h)}=\left\langle S^{z}\right\rangle,\left.\quad \frac{\mathrm{d}\left\langle S^{z}\right\rangle}{\mathrm{d}(-h \beta)}\right|_{X=\text { const }}=\left\langle S^{z} S^{z}\right\rangle_{\boldsymbol{q}=0}
$$

In the limit of vanishing fluctuations, our results go over into the ones obtained within the mean field approximation.

In the analytical form, the first term in the diagram series for the grand canonical potential (27) is equal to

$$
\frac{1}{2} \Xi X=\frac{1}{2} \frac{1}{\sqrt{2 \pi X}} \int_{-\infty}^{+\infty} \exp \left(-\frac{\xi^{2}}{2 X}\right) \xi b(y+\xi) \mathrm{d} \xi
$$

The bracketed diagram series can be presented as follows:

$$
\begin{aligned}
-\frac{1}{2}\left\{\frac{1}{2} \Xi^{2}\left(\frac{2}{N} \sum_{n, \boldsymbol{k}} \Lambda_{n}^{2} \tilde{t}_{n}(\boldsymbol{k})^{2}\right)^{2}-\frac{1}{3} \Xi^{3}\left(\frac{2}{N} \sum_{n, \boldsymbol{k}} \Lambda_{n}^{2} \tilde{t}_{n}(\boldsymbol{k})^{2}\right)^{3}+\cdots\right\}= \\
=-\frac{1}{2}\left\{\Xi \frac{2}{N} \sum_{n, \boldsymbol{k}} \Lambda_{n}^{2} \tilde{t}_{n}(\boldsymbol{k})^{2}-\ln \left(1+\Xi \frac{2}{N} \sum_{n, \boldsymbol{k}} \Lambda_{n}^{2} \tilde{t}_{n}(\boldsymbol{k})^{2}\right)\right\} .
\end{aligned}
$$

The remainder of the diagram in the series (27) can be written as

$$
-\left\{b(y)^{[1]} \frac{X}{2}+\frac{1}{2 !} b(y)^{[3]}\left(\frac{X}{2}\right)^{2}+\cdots\right\}=-\frac{1}{2} \int_{-\infty}^{+\infty}\left\{1-\operatorname{erf}\left(\frac{|\xi|}{\sqrt{2 X}}\right)\right\} \operatorname{sign}(\xi) b(y+\xi) \mathrm{d} \xi
$$


where we use the relation (26) and definition of the erf function. Finally, the diagram series for a grand canonical potential $\Omega$ can be written in the following analytical form:

$$
\begin{aligned}
\Omega= & \Omega_{\mathrm{MFA}}+\frac{1}{2} \frac{1}{\sqrt{2 \pi X}} \int_{-\infty}^{+\infty} \mathrm{e}^{-\frac{\xi^{2}}{2 X}} \xi b(y+\xi) \mathrm{d} \xi-\frac{1}{2} \int_{-\infty}^{+\infty}\left\{1-\operatorname{erf}\left(\frac{|\xi|}{\sqrt{2 X}}\right)\right\} \operatorname{sign}(\xi) b(y+\xi) \mathrm{d} \xi \\
& -\frac{1}{2} \Xi \frac{2}{N} \sum_{n, \boldsymbol{k}} \Lambda_{n}^{2} \tilde{t}_{n}(\boldsymbol{k})^{2}+\frac{1}{2} \ln \left(1+\Xi \frac{2}{N} \sum_{n, \boldsymbol{k}} \Lambda_{n}^{2} \tilde{t}_{n}(\boldsymbol{k})^{2}\right) .
\end{aligned}
$$

To sum over the Matsubara frequency, the relation (24) should be used in the limit of $\boldsymbol{k}^{\prime} \rightarrow \boldsymbol{k}$.

\section{Conclusion}

A consistent method is presented, which takes into account the corrections due to the gaussian fluctuation of self-consistent field, in order to calculate thermodynamic and correlation functions of the pseudospin-electron model with an effective interaction. The diagram series and corresponding formulae are obtained for the pseudospin mean value $\left\langle S^{z}\right\rangle$, for a pseudospin correlation function $\left\langle S^{z} S^{z}\right\rangle_{\boldsymbol{q}}$ as well as for the grand canonical potential $\Omega$. The possibility exists to investigate their behaviour under the changes of thermodynamical and model parameters. The parameter $X$ (22) (r.m.s. fluctuation of the mean field) is calculated by means of the self-consistent renormalization of the correlation function (12), (21). From the expression for a grand canonical potential, calculated within the scheme presented here, the equations for $\left\langle S^{z}\right\rangle$ and $\mathrm{X}$ parameters satisfying the stationary conditions (28) are obtained.

This approach can be used to investigate the role of gaussian fluctuations in the phase separation phenomena as well as in the transition into a modulated phase in PEM.

It should be noted that the analytical scheme presented in our paper can be easily reduced to the Onyszkiewicz type approach (which was successfully used in the case of spin models with direct spin interaction $[11,12]$ ) where the renormalization (20) is performed with the use of the simplest possible pseudospin correlation function involving gaussian fluctuations of the mean field:

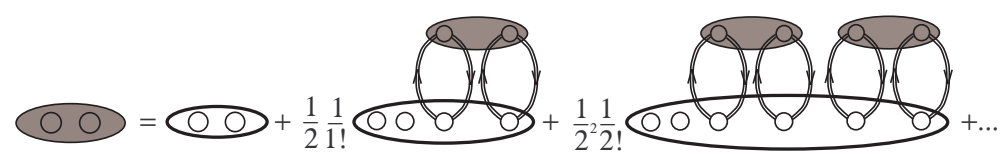

Within the framework of this approximation, grand canonical potential satisfies the stationary conditions (28) and can be written as:

$$
\Omega=\Omega_{\mathrm{MFA}}+\frac{1}{4} \Xi X-\frac{1}{2} \int_{-\infty}^{+\infty}\left\{1-\operatorname{erf}\left(\frac{|\xi|}{\sqrt{2 X}}\right)\right\} \operatorname{sign}(\xi) b(y+\xi) \mathrm{d} \xi
$$




$$
X=\Xi\left(\frac{2}{N} \sum_{\boldsymbol{q}, n} \Lambda_{n}^{2} \tilde{t}_{n}^{2}(\boldsymbol{k})\right)^{2}
$$

This method is more suitable for numerical calculations than the above presented but it takes into account the restricted class of diagrams.

\section{References}

1. Müller K.A. // Z. Phys. B, 1990, vol. 80, p. 193.

2. Matsushita E. // Phys. Rev. B, 1995, vol. 51, No. 24, p. 17332.

3. Stasyuk I.V., Shvaika A.M. // J. Phys. Studies, 1999, No. 3, p. 177.

4. Freericks J.K. // Phys. Rev. B, 1993, vol. 47, p. 9263.

5. Stasyuk I.V., Shvaika A.M., Tabunshchyk K.V. // Cond. Matt. Phys., 1999, vol. 2, No. 1(17), p. 109.

6. Stasyuk I.V., Shvaika A.M., Tabunshchyk K.V. // Acta Physica Polonica A, 2000, vol. 97 , No. 3, p. 411.

7. Stasyuk I.V., Shvaika A.M., Tabunshchyk K.V. // Ukrainian Journal of Physics, 2000, vol. 45 , No. $4-5$ p. 520.

8. Izyumov Yu.A., Letfulov B.M. // J. Phys.: Cond. Matter, 1990, vol. 2, p. 8905.

9. Izyumov Yu.A., Kassan-Ogly F.A., Skriabin Yu.N.// J. de Phys., 1971, 325, p. 1-87.

10. Izyumov Yu.A., Kassan-Ogly F.A., Skriabin Yu.N. Fields Methods in the Theory of Ferromagnets. Moscow, Mir, 1974 (in Russian).

11. Onyszkiewicz Z. // Phys. Lett., 1976, vol. 57A, p. 480.

12. Onyszkiewicz Z. // Phys. Lett., 1980, vol. 76A, p. 411. 


\title{
Самоузгоджене врахування гаусових флуктуацій у псевдоспін-електронній моделі
}

\author{
І.В.Стасюк, К.В.Табунщик \\ Інститут фізики конденсованих систем НАН України, \\ 79011 Львів, вул. Свєнціцького, 1 \\ Отримано 24 жовтня 2000 p.
}

В роботі пропонується аналітична самоузгоджена схема розрахунку термодинамічних і кореляційних функцій у псевдоспін-електронній моделі при відсутності кореляцій. Отримано аналітичні вирази для псевдоспінової кореляційної функції, середнього значення оператора псевдоспіну та термодинамічний потенціал в узагальненому наближенні хаотичних фаз при врахуванні поправок типу середнього поля, що виникають внаслідок ефективної взаємодії псевдоспінів через електрони провідності, а також при врахуванні гаусових флуктуацій середнього поля.

Ключові слова: псевдоспін-електронна модель, локальний ангармонізм, гаусові флуктуації

PACS: $71.10 . F d, 71.38 .+i, 77.80 . B h, 63.20 . R y$ 